\title{
Posterior reversible encephalopathy syndrome in a patient with diabetic ketonuria
}

\section{Sharma I', Banjade $\mathrm{P}^{2}$, Atthipalayam Chellamuthu $\mathrm{S}^{3}$, Saeed $\mathrm{F}^{4}$,Dallakoti $\mathrm{P}^{5}$}

'Ishwor Sharma, Department of Internal Medicine, ADK Hospital, Maldives; ${ }^{2}$ Prakash Banjade, Medical Officer, Department of Internal Medicine, Gan Regional Hospital, Maldives; ${ }^{3}$ Sasikumar Atthipalayam Chellamuthu, Department of Neurology, ADK Hospital, Maldives; ' ${ }^{2}$ aisal Saeed, Department of Quality improvement, Women's and Children's Health Network, SA Health, Adelaide, Australia; ${ }^{5}$ Prajut Dallakoti, Medical Officer, Gajuri Primary Health Center, Ministry of Health Government of Nepal, Nepal.

\begin{abstract}
Posterior reversible encephalopathy syndrome is a condition presenting with non-specific symptoms like nausea, vomiting and headache along with neurological manifestations like altered mental status, seizure, visual impairment and even coma. These symptoms are coupled with characteristic radiological findings of vasogenic edema in the bilateral parieto-occipital lobe which is usually reversible. We present here, a young 30 years old male, with dizziness, vomiting, generalized weakness, altered mental status with cortical blindness and focal and generalized tonic-clonic seizures in the background of first presentation of type 2 diabetes mellitus with ketonuria. Characteristic findings in Magnetic Resonance Imaging and reversal of the symptoms helped to reach the diagnosis of posterior reversible encephalopathy syndrome in our patient. The patient was discharged in stable condition after reversal of the symptoms and treatment of type 2 diabetes mellitus.
\end{abstract}

Key words: Cortical blindness; Diabetes mellitus; Posterior reversible encephalopathy syndrome.

DOI: https://doi.org/10.3126/jkmc.v8i4.32390

\section{INTRODUCTION}

ince its first recognition in 1996 by Henchey et al ${ }^{1}$, $\checkmark$ posterior reversible encephalopathy syndrome has been studied in different case studies and case series but the specific pathogenesis still remains unclear. It is a clinical radiologic syndrome with clinical presentation of nausea, vomiting, headache, altered mental status with or without focal neurological signs, seizure activity, visual impairment, paresis and even coma². Posterior reversible encephalopathy syndrome (PRES) may be associated with a number of medical conditions. Magnetic Resonance Imaging (MRI) of brain typically demonstrates similar evidence of vasogenic edema

Address for correspondence

Dr. Prakash Banjade

Medical Officer, Department of Internal Medicine

Gan Regional Hospital, Maldives

E-mail: ur.prakash25@gmail.com

Dr. Prakash Banjade: https://orcid.org/0000-0002-5089-9646

Ishwor Sharma: https://orcid.org/0000-0002-2813-2924

Prajut Dallakoti: https://orcid.org/0000-0003-0473-7782

Faisal Saeed: https://orcid.org/000-0002-4711-5572 in the subcortical white matter and cortex, which predominantly involves the bilateral parieto-occipital lobes $^{2}$. However, cytotoxic edema in or outside the massive vasogenic edema was also found in a relatively lower proportion of patients with PRES. Although most of the cases are reversible once the underlying cause is removed, the syndrome is not always reversible.

The exact incidence of PRES in not known. It is reported as early as two years to as old as 90 years of age. It may occur in diverse situations like hypertension, eclampsia, preeclampsia, use of immunosuppressive medications, autoimmune diseases, sepsis, shock, hemolytic uremic syndrome, thrombocytopenic syndromes, hypercalcemia, renal failure and post-transplantation. PRES is more common in females than males even when patients with eclampsia are excluded ${ }^{3}$.

We report a case of a 30-year-old male who presented with newly detected diabetes mellitus with ketonuria which was complicated by PRES. 


\section{CASE REPORT}

A 30-year-old male without any known co-morbidities presented to the emergency department with complaints of altered mental status of one day duration. He also had a history of dizziness for three days, several episodes of vomiting and generalized weakness but did not have fever. Family history was unremarkable for diabetes mellitus, hypertension or any autoimmune disease. He is a teetotaler and non-smoker.

On physical examination, he seemed disoriented, restless and mute. He had a blood pressure of $100 / 60 \mathrm{~mm}$ of mercury, pulse rate of 74 per minute, temperature of 36.7degree $C$, respiratory rate of 16 breaths per minute. The abdomen was soft and non-tender. Cardiovascular and respiratory system examinations were normal. Pupils were $2 \mathrm{~mm}$ size bilaterally and reactive to light. Neurological examination on admission was very limited as the patient was disoriented and restless and not obeying any command. However, he was moving all his limbs actively and plantars were flexor bilaterally.

Computed Tomography scan at admission was normal. Random blood sugar at admission was $277 \mathrm{mg} / \mathrm{dl}$ and urine ketone was positive. Arterial blood gas analysis did not show any acidosis. The patient had a recorded temperature of 38.0degree $\mathrm{C}$ after admission. There was no neck rigidity, Kernig's sign and Brudzunski's sign were negative.

One day after admission, patient complained of headache and vision loss. Vision assessment revealed diminution of vision of bilateral eyes to perception of light. Fundoscopic examination was normal. There was occasional jerky nystagmus in both eyes. Rests of the cranial nerve examination were normal. Power was 5/5 across all major joints of upper and lower limbs. Sensory examination was normal. Cerebellar signs were intact. Vision started to improve from third day of admission with finger counting on the third day. On the fourth day of admission, he initially developed one episode of focal seizure and another episode of generalized tonic-clonic seizure that occurred six hours apart. Blood pressure immediately after generalized tonic-clonic seizure was elevated to $152 / 82 \mathrm{~mm}$ of mercury. Other vital signs were within normal limits. The patient regained consciousness after seizure but was complaining of headache. Detailed neurological examination did not reveal any focal neurological deficit. The case was reported on 2019/04/01 and the treatment was completed by 2019/06/01.

\section{INVESTIGATIONS}

Lab results revealed significant leukocytosis with $16,300 / \mathrm{cu} \mathrm{mm}$, platelets - 280,000/cumm, haemoglobin -14.7 gm\%/dl, C-reactive protein - 4.08mg/dl, random blood sugar - $255 \mathrm{mg} / \mathrm{dl}$, fasting blood sugar-155mg/ dl, HbA1c-8.6\%, Alanine aminotransferase $-76 \mathrm{U} / \mathrm{L}$, Aspartate transaminase-36U/L, creatinine - $0.54 \mathrm{mg} /$ $\mathrm{dl}$ and urea-20mg/dl. Electrolytes were normal. Urine ketones were $3+$. Arterialblood gas analysis showed - $\mathrm{pH}-4.42$, partial pressure of carbondioxide $\left(\mathrm{PCO}_{2}\right)$ -30.0, partial pressure of oxygen $\left(\mathrm{PO}_{2}\right)-96$, bicarbonate $\left(\mathrm{HCO}_{3}\right)-$ 21.2, anion gap-19.9.Prothrombin time/

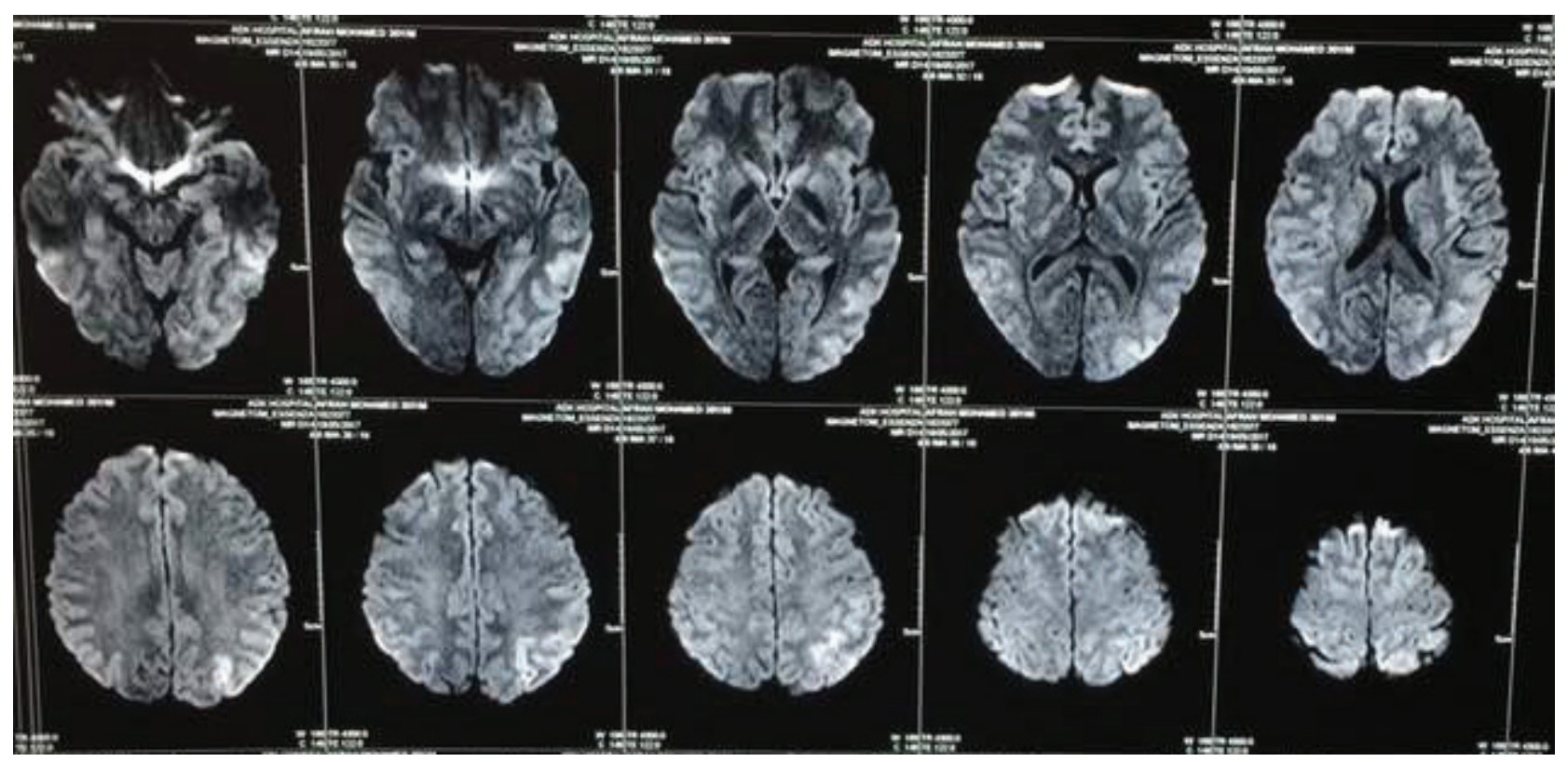

Figure 1: MRI brain 
International normalized ratio (PT/INR), activated partial thromboplastin time (APTT), thyroid function tests (TFT) were normal. Viral serologyfor hepatitis B, C andHuman immunodeficiency virus(HIV) were non-reactive. Chest $\mathrm{x}$-ray was normal. CT head was normal. Cerebrospinal fluid (CSF) analysis was also normal with clear appearance, total cell count - 6/cumm, polymorphs$17 \%$, mononuclear cells $-83 \%$, protein $-31.33 \mathrm{mg} / \mathrm{dl}$, and glucose- $129 \mathrm{mg} / \mathrm{dl}$. Magnetic Resonance Venography (MRV) of brain showed normal study. MRI brain showed focal areas of restricted diffusion in the left high parietal lobe and occipital lobe with reversal on the Apparent Diffusion Coefficient (ADC) map and mild gyral edema in both cerebral hemispheres with signal lengthening of sulcal content on FLAIR images consistent with PRES (Figure 1).

\section{DIFFERENTIAL DIAGNOSIS}

As the patient presented with altered mental status within one day with vomiting and generalized weakness,a working diagnosis of meningoencephalitis was considered with the following differential diagnosis: 1. Cerebrovascular accident 2. Cortical venous thrombosis 3. PRES. Lumbar puncture and CSF analysis ruled out meningoencephalitis. A normal fundus examination with normal MRV of brain ruled out cortical venous thrombosis. The reversibility of symptoms along with the characteristic MRI findings were suggestive of PRES.

\section{TREATMENT}

Patient was admitted to High Dependency Unit (HDU) with the diagnosis of newly diagnosed diabetes mellitus with meningoencepahlitis. Ceftriaxone was started at twograms intravenous(IV) two times a day(BD). Basal (Glargine) and bolus (rapid acting regular insulin) regimen of insulin was started for uncontrolled diabetes mellitus. Clonazepam $0.5 \mathrm{mg}$ BD for three days followed by $0.5 \mathrm{mg}$ once daily (OD) for one week was given. Levetiracetam $250 \mathrm{mg}$ BD was started and continued and tapered off in six weeks. After the CSF report turned out to be normal and MRI was consistent with PRES, ceftriaxone was stopped.

\section{OUTCOME AND FOLLOW-UP}

The patient's altered mental status improved on second day. His headache and vision impairment improved gradually over the course of a week and during discharge his vision was 6/6. He was seizure free after adding levetiracetam. After observing in HDU for four days, he was transferred to a private room and was discharged on the eighth day of admission. He was symptom-free at the time of discharge.

\section{DISCUSSION}

PRES is a clinico-radiologic entity with typical MR imaging showing predominant vasogenic edema and occasional cytotoxic edema of white matter and cortex affecting typically the bilateral parieto-occipital lobes ${ }^{2}$. The syndrome is not always reversible although most cases are reversible. The exact incidence of PRES is unknown. It can occur in any age group. Case reports from various parts of the world range from age two to ninety years. It is more common in women ${ }^{3}$.

PRES can develop due to a myriad of clinical conditions. To mention some, the most commonly mentioned associated conditions are hypertensive encephalopathy, acute or chronic renal diseases, thrombotic thrombocytopenic purpura, hemolytic uremic syndrome, eclampsia, vasculitis, sepsis, immunosuppressive, immunomodulatory and chemotherapeutic drugs, porphyria, hypercalcemia, hypermagnesemia, blood transfusion and contrast media exposure. Diabetes mellitus has also been described as a complicating factor in $\mathrm{PRES}^{5}$. Our patient did not have any of the aforementioned associated conditions except diabetes mellitus. However, genetic analysis for $3243 \mathrm{~A} \rightarrow \mathrm{G}$ mutation was not done, which could be one of the etiologies when diabetes is present along with PRES.

The presenting features of PRES are headache, encephalopathy, visual disturbances and seizures. Headache may be continuous, diffuse and intensity ranging from moderate to severe ${ }^{3}$. Decreased consciousness may manifest as drowsiness or stupor and coma at its worse. Visual abnormalities may present in the form of hemianopia, visual neglect, visual hallucinations and cortical blindness. There may even be denial of blindness also known as Anton's Syndrome. Seizures may be focal, generalized tonic-clonicor even status epilepticus. Fundoscopic exam is usually normal. There may be some focal neurological symptoms and signs.

Diagnosis of PRES is made on the basis of clinical syndrome, characteristic MRI findings and exclusion of other differential diagnosis. There are no specific diagnostic criteria for the diagnosis of PRES. Differential diagnoses includes stroke, meningoencephalitis, encephalitis, cerebral venous thrombosis, toxic or metabolic encephalopathies, demyelinating disorders and vasculitis. Early imaging helps to clinch the diagnosis. MRI findings are typically symmetrical white matter edema in the posterior cerebral hemisphere. There may be edema in the gray matter and basal gangliaas well. The lesions appear hypointense on T1- 
weighted imaging and hyperintense on T2-weighted imaging MRI. Atypical imaging includes contrast enhancement, hemorrhage and restricted diffusion on MRI. These abnormalities partially or completely resolve on follow up imaging suggesting that it is not an infarct. PRES involving the occipital lobe spare the calcarine and paramedian occipital lobe, distinguishing PRES from bilateral cerebral infarctions ${ }^{6,7}$.

The management recommendations for PRES are basically symptomatic and treatment of underlying cause. High clinical suspicion is necessary in appropriate clinical setting because early identification can help to

\section{REFERENCES}

1. Hinchey J, Chaves C, Appignani B, Breen J, Pao L, Wang $A$, et al. A reversible posterior leukoencephalopathy syndrome. The New England journal of medicine. 1996;334(8):494-500. [DOI]

2. Gao B, Yu BX, Li RS, Zhang G, Xie HZ, Liu FL, et al. Cytotoxic Edema in Posterior Reversible Encephalopathy Syndrome: Correlation of MRI Features with Serum Albumin Levels. AJNR American journal of neuroradiology. 2015;36(10):1884-9. [DOI]

3. Rijal JP, Giri S, Dawadi S, Dahal KV. Posterior reversible encephalopathy syndrome (PRES) in a patient with late postpartum eclampsia. BMJ case reports. 2014. [DOI]

4. Bartynski WS, Boardman JF, Zeigler ZR, Shadduck RK, Lister J.Posterior reversibleencephalopathy syndrome in infection, sepsis, and shock. AJNR American journal of neuroradiology. 2006;27(10):2179-90.

5. Jones R, Redler K, Witherick J, Fuller G, Mahajan T, Wakerley BR. Posterior reversible encephalopathy syndrome complicating diabetic ketoacidosis; an important treatable complication. Pediatric diabetes. 2017;18(2):159-62. [DOI] treat the cause and ensure reversibility ${ }^{3,7,8}$. Although PRES is usually benign, some cases may complicate with permanent neurologic deficits, secondary intracerebral hemorrhage or infarction and even death ${ }^{9}$.Radiologic improvement lags behind clinical improvement. Recurrence may also occur in less than $8 \%$ of $\operatorname{cases}^{10}$.

\section{CONCLUSION}

Our case highlights the need to consider PRES in uncontrolled diabetes mellitus with seizures and other neurologic findings when the MRI findings are characteristic. Awareness of PRES and its radiologic findings is essential to avoid misdiagnosis and delay in treatment.

6. Naqi R, Ahsan H, Azeemuddin M. Posterior reversible encephalopathy syndrome: a case series in patients with eclampsia. JPMA The Journal of the Pakistan Medical Association. 2010;60(5):394-7.

7. McKinney AM, Short J, Truwit CL, McKinney ZJ, Kozak OS, SantaCruz KS, et al. Posterior reversible encephalopathy syndrome: incidence of atypical regions of involvement and imaging findings. AJR American journal of roentgenology. 2007;189(4):90412. [DOI]

8. Servillo G, Striano P, Striano S, Tortora F, Boccella P, De Robertis $E$, et al. Posterior reversible encephalopathy syndrome (PRES) in critically ill obstetric patients. Intensive care medicine. 2003;29(12):2323-6. [DOI]

9. Narbone MC, Musolino R, Granata F, Mazzu I, Abbate $M$, Ferlazzo E. PRES: posterior or potentially reversible encephalopathy syndrome? Neurological sciences : official journal of the Italian Neurological Society and of the Italian Society of Clinical Neurophysiology. 2006;27(3):187-9. [DOI]

10. Roth C, Ferbert A. Posterior reversible encephalopathy syndrome: long-term follow-up. Journal of neurology, neurosurgery, and psychiatry. 2010;81(7):773-7. [DOI] 